\title{
Hybrid beat in nuclear forward scattering of synchrotron radiation
}

\author{
Yu. V. Shvyd'ko \\ II. Institut für Experimentalphysik, Universität Hamburg, D-22761 Hamburg, Germany \\ U. van Bürck, W. Potzel, and P. Schindelmann \\ Physik-Department E15, Technische Universität München, D-85748 Garching, Germany \\ E. Gerdau and O. Leupold \\ II. Institut für Experimentalphysik, Universität Hamburg, D-22761 Hamburg, Germany \\ J. Metge \\ European Synchrotron Radiation Facility, Boîte Postale 220, F-38043 Grenoble, France \\ H. D. Rüter \\ II. Institut für Experimentalphysik, Universität Hamburg, D-22761 Hamburg, Germany
}

G. V. Smirnov

R.R.C. “Kurchatov Institute,'” 123182 Moscow, Russia

(Received 7 July 1997)

\begin{abstract}
Asymmetric nuclear-resonance broadening, as originating, e.g., from magnetic-hyperfine-field distributions in magnetic alloys, has strong effects on the time evolution of nuclear forward scattering of synchrotron radiation. In thin samples of an Invar alloy, resonance broadening and the resulting dephasing in time cause a fast decay of the coherent scattering signal. In thick samples, the asymmetry of the broadening strongly affects dynamical scattering. Quantum beat and dynamical beat blend into a fast hybrid beat with thickness dependent period and field distribution sensitive modulation. [S0163-1829(98)01405-2]
\end{abstract}

\section{INTRODUCTION}

The advent of intense pulsed sources of synchrotron radiation (SR) made the observation of pure nuclear Bragg scattering of SR (Ref. 1) possible providing the basis for Mössbauer spectroscopy in the time domain (for a review see, e.g., Refs. 2,3). But only the introduction of meVresolution $\mathrm{X}$-ray monochromators made the observation of nuclear forward scattering (NFS) of SR feasible ${ }^{4}$ and has thus opened a broad field of applications for time-domain spectroscopy.

In conventional Mössbauer spectroscopy the dependence of nuclear absorption on the energy of the incident radiation is measured. The recorded signal in this case presents the incoherent sum of the spectral components of the transmitted radiation. In time-domain spectroscopy, by contrast, the scattering spectrum of nuclei excited by a pulse of white SR is measured in the time domain, where the response is formed by the coherent sum of the spectral components of the scattered radiation. This results in important interference effects specific for time-domain Mössbauer spectroscopy.

Interference of radiation scattered by nuclear resonances of different energies, arising, e.g., due to hyperfine interactions, leads to quantum beats (QB's) ${ }^{5,6}$ Henceforth we shall refer to the interference of this type as inter-resonance interference. In optically thick targets, multiple nuclear scattering makes the spectral components below and above resonance to dominate. ${ }^{3}$ The interference of these two groups of components (intraresonance interference) in optically thick tar- gets yields dynamical beats (DB's) in the time response of NFS. $^{7-10}$ The coherent combination of these two beat phenomena makes the time dependence of NFS both complex in structure and rich in information.

This happens already for rather simple systems, where the nuclei at their sites experience definite values of the magnetic-hyperfine fields, electric field gradients, and electron densities. Nowadays, however, interest has shifted towards more complex systems, which are characterized by distributions of the hyperfine-field parameters. Such distributions are usually directly connected with the nature of the sample under study, as for example magnetic-field distributions in intermetallic alloys, but they might also be produced by the experimental technique, e.g., by pressure gradients hardly avoidable in high-pressure cells.

The present work was aimed at studying the influence of inhomogeneous nuclear-resonance broadening on the time dependence of NFS. We were able to reveal pronounced effects due to resonance broadening. In particular, due to an asymmetry of the broadening, DB and QB blend into a fast hybrid beat. As an example we investigated the effects arising from a distribution of hyperfine fields in magnetic Invar alloys. Since this was, to our knowledge, the first study in this field, we were primarily interested in revealing and understanding the basic effects, rather than details of our actual samples.

In Sec. II basic aspects of the NFS theory are presented. In particular, the influence of inhomogeneous nuclearresonance broadening on the time dependence of NFS is dis- 
cussed. In Sec. III the measurement procedure and the samples under study are described. The results of the measurements of NFS by thin and thick samples of iron and Invar are given in Sec. IV and discussed in Sec. V.

\section{BASIC ASPECTS OF NFS THEORY}

\section{A. General formulas}

The propagation of a radiation field $\boldsymbol{E}(\boldsymbol{r}, t)$ through a resonant medium (in form of a plate of thickness $L$ ) can be described in space and time by the wave equation ${ }^{9,11,12}$

$$
\begin{gathered}
\frac{d E^{s}(\zeta, \tau)}{d \zeta}=-\int_{-\infty}^{\tau} d \tau^{\prime} K^{s}\left(\tau-\tau^{\prime}\right) E^{s}\left(\zeta, \tau^{\prime}\right), \\
K^{s}(\tau)=\sum_{l} \xi_{l}^{s} \exp \left[i \frac{E-E_{l}}{\Gamma_{0}} \tau-\frac{\tau}{2}\right], \\
\zeta=z /(L \sin \Theta) ; \quad \tau=\Gamma_{0} t / \hbar .
\end{gathered}
$$

Here $E^{s}(\zeta, \tau)$ is the $s$-polarization component of the amplitude of the radiation field $\boldsymbol{E}(\boldsymbol{r}, t)=\boldsymbol{E}(\zeta, t) \exp [i \boldsymbol{k r}-\mathrm{i} E t / \hbar]$ which varies slowly compared to the variation of the exponent; $z$ is the spatial coordinate in the direction of the internal normal to the entrance surface; the index $l \equiv\{m \Leftrightarrow M, j\}$ is used to numerate both the different nuclear transitions between ground and excited states and different nuclear sites; $\Theta$ is the angle between incident beam and surface. For simplicity we assume that the nuclei experience only magnetic hyperfine interactions and that the magnetic quantization axis $\boldsymbol{n}$ is perpendicular to the wave vector $\boldsymbol{k}$ at each nuclear site. Under these assumptions the energy of the nuclear transition $l$ is given by

$$
\begin{gathered}
E_{l}=E_{0}+\hbar \Omega_{m M}\left(B_{j}\right), \\
\hbar \Omega_{m M}\left(B_{j}\right)=\left(m \mu_{g} / I_{g}-M \mu_{e} / I_{e}\right) B_{j},
\end{gathered}
$$

where $E_{0}$ is the energy of the nuclear transition between the ground and excited state in the absence of hyperfine interactions, $\mu_{g, e}$ are nuclear magnetic moments, $I_{g, e}$ are nuclear spins and $m, M$ are magnetic quantum numbers in the ground and excited states, respectively, $B_{j}$ is the value of the magnetic hyperfine field at the nuclear site $j$.

The value

$$
\xi_{l}^{s}=\frac{1}{4} k L g_{l}^{s}
$$

is a partial effective resonance thickness parameter for the specific transition $l$ and polarization $s$. Here

$$
\begin{gathered}
g_{l}^{s}=g_{j}\left(\begin{array}{ccc}
I_{g} & 1 & I_{e} \\
-m & m-M & M
\end{array}\right)^{2} 3 P_{m-M}^{s}, \\
g_{j}=\frac{\sigma_{R} N_{0}}{k} w_{j} f_{\mathrm{LM}} ; \quad \sigma_{R}=\frac{4 \pi}{k^{2}} \frac{2 I_{e}+1}{2\left(2 I_{g}+1\right)} \frac{1}{1+\alpha}, \\
P_{m-M}^{s}= \begin{cases}\left(\mathbf{n h}^{s}\right)^{2}, & m-M=0, \\
\frac{1}{2}\left[1-\left(\mathbf{n h}^{s}\right)^{2}\right], & m-M= \pm 1,\end{cases}
\end{gathered}
$$

where $(\cdots)$ is the $3 j$ symbol, $N_{0}$ is the number of resonant nuclei per unit volume, $w_{j}$ is a weight factor of the nuclear site $j, f_{\mathrm{LM}}$ is the Lamb-Mössbauer factor and $\alpha$ is the internal conversion coefficient, and $\boldsymbol{h}^{s}$ is the magnetic polarization vector of the radiation. In defining the polarization factor $P_{m-M}^{s}$ we have assumed in accordance with our experimental conditions that $\boldsymbol{n} \perp \boldsymbol{k}$ holds at each nuclear site. Two types of solutions of the wave equation (1) are of interest for us.

The first one is the solution in energy space which describes the transmission of monochromatic incident radiation with energy $E$ through the sample plate. The boundary condition for the radiation amplitude at the entrance surface is then given by $E^{s}(0, t)=\mathcal{E}^{s}$, where $\mathcal{E}^{s}$ is the amplitude of the incident radiation. The solution of Eq. (1) is therefore of the steady-state type and the transmission amplitude $R^{s}(E)$ $=E^{s}(L, t) / E^{s}(0, t)$ equals

$$
R^{s}(E)=\exp \left(-i \sum_{l} \frac{\xi_{l}^{s} \Gamma_{0}}{E-E_{l}+i \Gamma_{0} / 2}\right) .
$$

The second type of solutions of interest are time responses of the nuclear-resonance ensemble onto the instantaneous excitation by a short radiation pulse. The boundary condition in this case reads $E^{s}(0, t)=\mathcal{E}^{s} \delta(t)$. The general solution of the wave equation can be presented as a power series of $\zeta:^{12}$

$$
E^{s}(\zeta, \tau)=A_{0}^{s}(\tau)+\sum_{p=1}^{\infty} \frac{(-\zeta)^{p}}{p !} A_{p}^{s}(\tau),
$$

with the functions $A_{p}^{s}(\tau)$ determined according to the following recursion relations:

$$
\begin{gathered}
A_{0}^{s}(\tau)=\mathcal{E}^{s} \delta(\tau), \\
A_{1}^{s}(\tau)=\mathcal{E}^{s} K^{s}(\tau) \theta(\tau), \\
A_{p}^{s}(\tau)=\int_{-\infty}^{\tau} d \tau^{\prime} K^{s}\left(\tau-\tau^{\prime}\right) A_{p-1}^{s}\left(\tau^{\prime}\right),
\end{gathered}
$$

which one obtains by inserting Eq. (10) into Eq. (1) and taking into account the boundary condition. $\theta(t)$ is the unit step function.

We shall present examples of a few particular solutions. First we consider the case of a thin nuclear resonance scatterer, i.e., when $\xi^{s}<1$. In this case the series in Eq. (10) may be cut off after the term with $p=1$. Then using Eqs. (12), (2) the solution for the radiation field at the exit surface of the sample, i.e., when $z=L$, at times $t>0$ reads

$$
E^{s}(L, t) \propto \exp \left(-\frac{\Gamma_{0}}{2 \hbar} t\right) \sum_{l} \xi_{l}^{s} \exp \left(-\frac{i}{\hbar} E_{l} t\right) .
$$

Thus it is seen that the nuclear system responds at the frequencies of the excited nuclear transitions. The time spectrum, which is $I(t) \propto\left|E^{s}(L, t)\right|^{2}$, contains a sum of sinusoidal functions, producing periodic modulations of the signal at the difference frequencies $\left(E_{l^{\prime}}-E_{l^{\prime \prime}}\right) / \hbar$. These modulations have been called quantum beats. ${ }^{5,6}$ 
In the next example we consider a sample of arbitrary thickness, however, with an unsplit nuclear resonance, i.e., $E_{l}=E_{0}$ for all $l$. In this case the summation over $l$ in Eq. (2) yields a single and polarization independent effective resonance thickness value $\xi=\Sigma_{l, s} \xi_{l}^{s}$, which corresponds to $T / 4$, where $T$ is the effective thickness used in conventional Mössbauer spectroscopy. All coefficients in Eq. (10) can then be calculated explicitly $A_{p}(\tau)=(\xi)^{p} \tau^{p-1} /(p-1)$ !. The summation of the series in Eq. (10) results (for $t>0$ ) in

$$
E(L, t) \propto-\xi \exp \left(-\frac{i}{\hbar} E_{0} t-\frac{\Gamma_{0}}{2 \hbar} t\right) \frac{\mathrm{J}_{1}(2 \sqrt{\xi \tau})}{\sqrt{\xi \tau}} .
$$

This expression, where $\mathrm{J}_{1}$ is the Bessel function of first kind and order one, was first obtained in Ref. 8. The Bessel modulation of the nuclear response to the prompt excitation is an effect of multiple coherent nuclear scattering, which can be observed in samples of significant thickness, ${ }^{4,10}$ and which is now often referred to as dynamical beat. A related Bessel modulation of the nuclear signal in time due to sample thickness was also observed with Mössbauer radioactive sources by using delayed coincidence ${ }^{7}$ and fast shutter ${ }^{9}$ techniques. In thick samples $(\xi \gg 1)$ the Bessel function describes a faster decay as compared to the natural decay $\exp \left(-\Gamma_{0} t / \hbar\right)$ in case of a thin sample [see Eq. (14)]. This effect is often referred to as coherent speed-up of the decay in NFS. ${ }^{8,9,13,10}$

If the nuclear transition energies $E_{l}$ are well separated then even in case of a thick sample one can use an approximate solution ${ }^{9}$

$$
E^{s}(L, t) \propto-\sum_{l} \xi_{l}^{s} \exp \left(-\frac{i}{\hbar} E_{l} t-\frac{\Gamma_{0}}{2 \hbar} t\right) \frac{\mathrm{J}_{1}\left(2 \sqrt{\xi_{l}^{s} \tau}\right)}{\sqrt{\xi_{l}^{s} \tau}} .
$$

which comprises both QB's and DB's.

In the general case the hyperfine splitting is not zero, the transition energies are not well separated, and the sample is not thin, then the time response can be calculated only numerically. The results of the numerical calculations presented in this paper were performed by using Eqs. (1)-(13) implemented in the computer program MOTIF. ${ }^{14}$

\section{B. Application to inhomogeneous nuclear-resonance broadening}

An example for NFS in the case of a nonbroadened nuclear resonance is given in Fig. 1(a). The figure shows a calculated time spectrum $I(t)$ of NFS by a magnetized iron sample under conditions where the two equivalent $m-M$ $=0$ nuclear transitions are excited. It is assumed that the magnetic hyperfine field has a definite value of about $33 \mathrm{~T}$ (see inset). The time spectrum reflects the inter-resonance interference between the scattered radiation components by a fast $\mathrm{QB}$ of period $\sim 8 \mathrm{~ns}$. The intraresonance interference leads to a pronounced DB modulation with minima at 77 and $260 \mathrm{~ns}$. This case corresponds to the approximation given in Eq. (16).

Which changes in the NFS time spectra can be expected if the nuclear resonances experience inhomogeneous broadening? The answer certainly depends on the origin of the broadening. It can be of static origin due to, e.g., hyperfine-

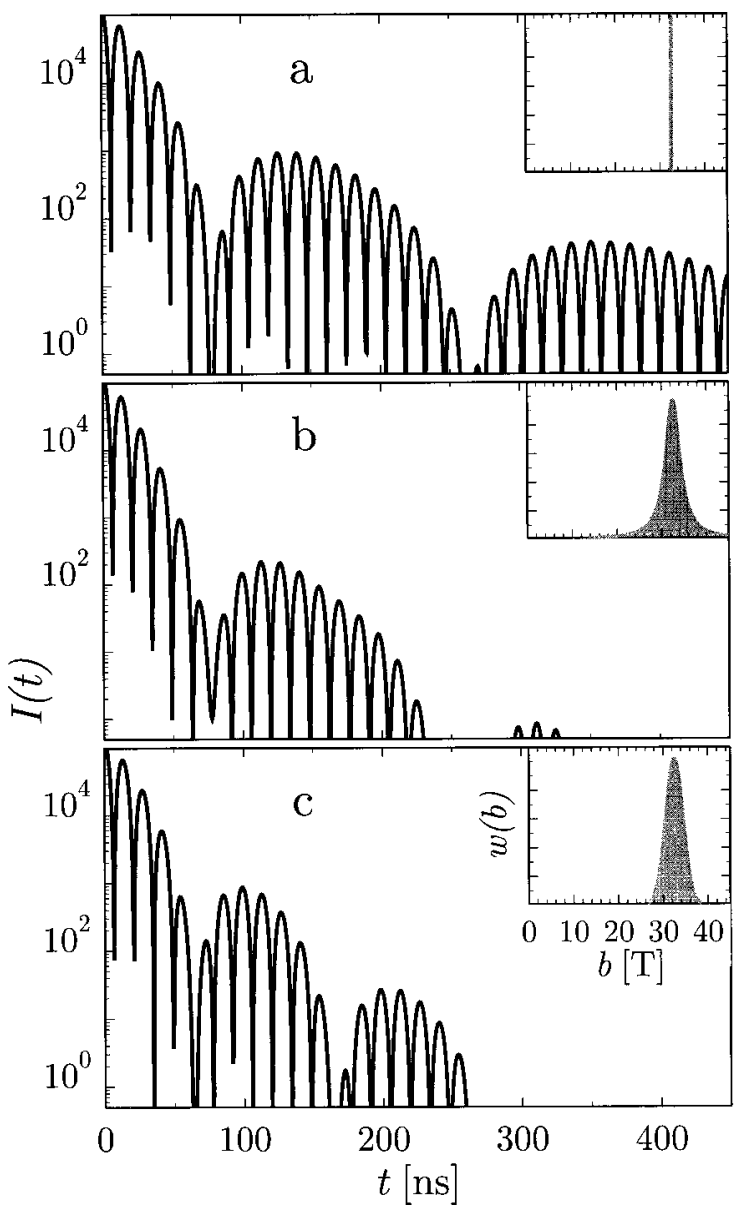

FIG. 1. Time evolution of NFS and magnetic hyperfine field profile (inset) for a nuclear scatterer equivalent to a magnetized ${ }^{57} \mathrm{Fe}$ foil of $3.5 \mu \mathrm{m}$ thickness, calculated for different field distributions: definite value of magnetic field (a), symmetrical distributions of Lorentzian shape (b), and of Gaussian shape (c).

field distributions, or it can be of dynamic origin due, e.g., to relaxation, diffusion, etc. These cases can be described with the same equation (1), however, with the kernel $K^{s}(\tau)$ averaged over the corresponding statistical distributions. ${ }^{12}$

We give two simple examples. Let us first consider a model, where at different nuclear sites the magnetic hyperfine field takes different values, and these values have a Lorentzian distribution around $B$ with a full width at half maximum given by $\Delta B$. We shall replace the weights in Eq. (7) by the distribution function

$$
w(b)=\frac{\Delta B}{2 \pi} \frac{1}{(b-B)^{2}+(\Delta B / 2)^{2}}
$$

and replace the summation over the site number $j$ in Eq. (2) by integration over $b$ between infinite limits. Performing the integration we obtain

$$
\begin{aligned}
& K^{s}(\tau)=\sum_{m M} \xi_{m M}^{s} \exp \left[i \frac{E-E_{m M}}{\Gamma_{0}} \tau-\frac{\tau}{2}\left(1+\gamma_{m M}\right)\right], \\
& E_{m M}=E_{0}+\hbar \Omega_{m M}(B) ; \quad \gamma_{m M}=\hbar \Omega_{m M}(\Delta B) / \Gamma_{0} .
\end{aligned}
$$

The kernel (17) looks just the same as the original one [Eq. (2)] with the only exception that the exponential damping 
constant is now $\left(1+\gamma_{m M}\right) / 2$ instead of $1 / 2$. This means that all solutions for the time dependences of NFS, such as Eqs. (14) -(16), are also valid in this case with the only modification that the exponential damping factor $\Gamma_{0}$ should be replaced by $\left(1+\gamma_{m M}\right) \Gamma_{0}$. In agreement with Refs. 15-17 one can thus conclude that a Lorentzian field distribution does not change the positions of the QB and DB minima. The essential influence of the Lorentzian inhomogeneous broadening is a faster decay of the NFS signal. These features become obvious from a comparison of Figs. 1(a) and 1(b).

By contrast, a Gaussian distribution of the magnetic hyperfine fields

$$
w(b)=\frac{1}{\Delta B \sqrt{2 \pi}} \exp \left[-\frac{(b-B)^{2}}{2(\Delta B)^{2}}\right]
$$

leads to more significant changes in the kernel:

$$
K^{s}(\tau)=\sum_{m M} \xi_{m M}^{s} \exp \left[i \frac{E-E_{m M}}{\Gamma_{0}} \tau-\frac{\tau}{2}-\frac{\tau^{2} \gamma_{m M}^{2}}{2}\right] .
$$

The solution of the wave equation with this kernel [Fig. 1(c)] yields the same QB modulation as in Figs. 1(a) and 1(b). However, the DB modulation is now changed drastically. In particular, the DB minima are now shifted to earlier times.

These two simple examples clearly demonstrate that the time dependence of NFS and in particular the DB and QB are very sensitive to the detailed form of the inhomogeneous broadening. Experimental studies of this question and further theoretical discussion are presented in the following sections.

\section{SAMPLES AND MEASUREMENTS}

An Invar alloy of composition $\mathrm{Fe}_{65} \mathrm{Ni}_{35}$, enriched to $95 \%$ in ${ }^{57} \mathrm{Fe}$, was used as sample material. Such alloys are ferromagnetic, and are considered in Mössbauer spectroscopy as classical, but still disputed ${ }^{18-20}$ examples for predominantly inhomogeneous resonance broadening due to static magnetic hyperfine field distributions. At room temperature they exhibit a broadening of several natural linewidths $\Gamma_{0} \cdot{ }^{18}$ Two different foils of 10 and $40 \mu \mathrm{m}$ thickness were prepared. A piece of the $10 \mu \mathrm{m}$ foil was used later to roll foils of $\sim 6$ and $1 \mu \mathrm{m}$ thickness. Reference measurements were performed with foils of pure iron metal, enriched to $95 \%$ in ${ }^{57} \mathrm{Fe}$, of corresponding thicknesses.

The foils were roughly characterized by conventional Mössbauer spectroscopy at room temperature, using unpolarized radiation from an intense ${ }^{57} \mathrm{CoRh}$ source. Figure 2 depicts the spectra of a $1 \mu \mathrm{m}$ iron reference foil, and of Invar foils of $0.7,6$, and $40 \mu \mathrm{m}$ thickness as used in the experiments. The foils were placed in an external magnetic field of $\sim 0.1 \mathrm{~T}$ in order to orient the hyperfine fields perpendicular to the beam direction. The thinnest Invar foil exhibits a magnetic hyperfine splitting, which is $\sim 80 \%$ of that of the iron foil, and a resonance broadening, which is $\sim 8 \Gamma_{0}$ for the $M-m=0$ transitions. In all spectra except the one in Fig. 2(b) broadening effects due to resonance absorption in optically thick samples prevail.

Studies of the time dependence of NFS by these samples ${ }^{21}$ were performed at HASYLAB (Hamburg) at room tempera-

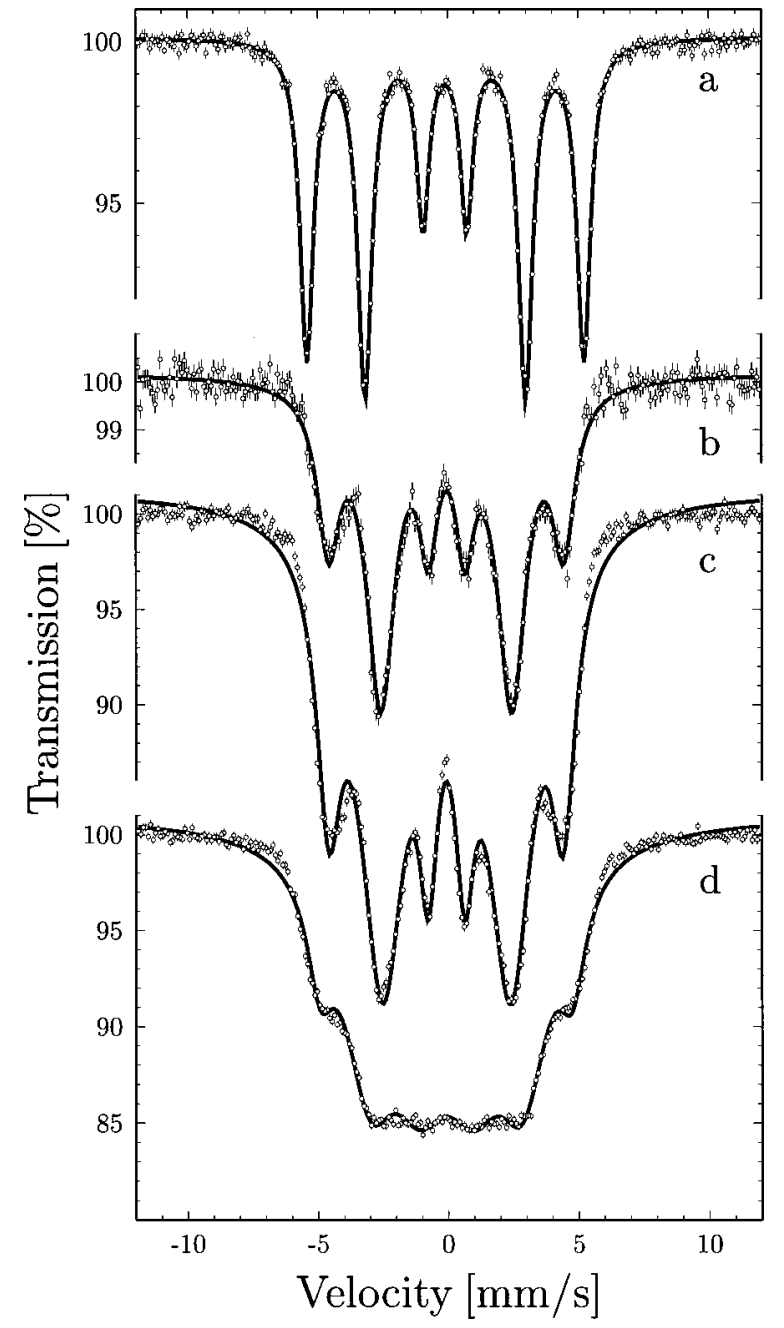

FIG. 2. Mössbauer spectra of a $1 \mu \mathrm{m}$ iron metal foil (a) and of Invar foils of $0.7 \mu \mathrm{m}$ (b), $6 \mu \mathrm{m}$ (c) and $40 \mu \mathrm{m}$, (d) thickness. The foils were mounted in a weak magnetic field perpendicular to the beam direction. The solid lines are guides to the eye based on the superposition of six independent Lorentzians.

ture at the bending magnet beam-line F4 (Ref. 22) and at liquid $\mathrm{He}$ temperature in a cryostat at the wiggler beam-line BW4. ${ }^{23}$ The SR was monochromatized to $6 \mathrm{meV}$ by a highresolution monochromator. ${ }^{24}$ The SR transmitted through the sample was recorded by an avalanche photo diode. ${ }^{25}$ The samples were placed in magnetic fields, which aligned the hyperfine fields perpendicular to the beam. Due to the linear polarization of SR (magnetic-field vector perpendicular to the plane of the storage ring) and the pure $M 1$ character of the $14.4 \mathrm{keV}$ nuclear resonance of ${ }^{57} \mathrm{Fe}$, either the two nuclear transitions with $M-m=0$ were excited when the external magnetic field was oriented vertically, or the four nuclear transitions with $M-m= \pm 1$ when the field was oriented horizontally. At room temperature a field of $\sim 0.13 \mathrm{~T}$ produced by a permanent magnet was applied, and in the cryostat at liquid He temperature a field of $1 \mathrm{~T}$ was provided by superconducting coils.

In the experiments, at first NFS by iron foils was measured in order to demonstrate as a reference the characteristic patterns produced by interresonance and intraresonance interference for a material without inhomogeneous broadening. 


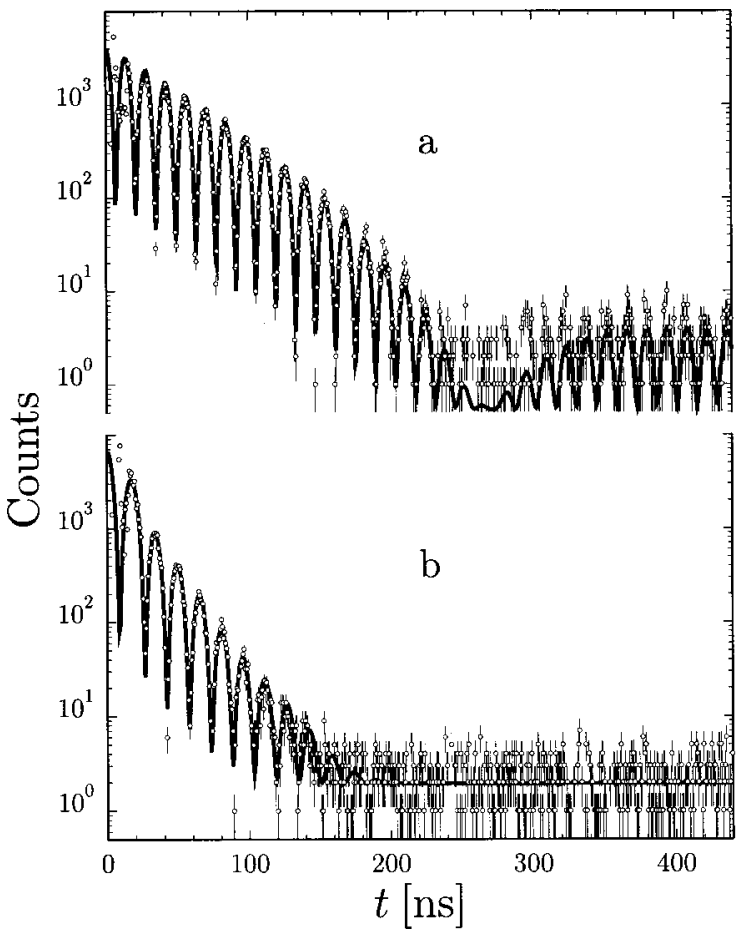

FIG. 3. Time evolution of NFS of SR for a $1 \mu \mathrm{m}$ iron metal foil (a) and a $1.2 \mu \mathrm{m}$ Invar foil (b) in a vertical magnetic field of $\sim 0.13$ $\mathrm{T}$. The solid lines are fits using the NFS theory.

The same measurements were then performed using the Invar foils as an example for a material with inhomogeneous broadening.

\section{RESULTS}

\section{A. Thin samples}

As discussed in Sec. II, inhomogeneous broadening is expected to lead to a faster decay of the NFS signal. In order to distinguish this effect from the coherent speed-up, which arises in optically thick samples, it was important to use samples as thin as could be tolerated for intensity reasons.

Figure 3(a) shows the time dependence of NFS by a $1 \mu \mathrm{m}$ iron foil placed in a vertical magnetic field. Only the two transitions with $M-m=0$ were excited, yielding a QB with a single period of $2 \pi \hbar / \delta E=14 \mathrm{~ns}$ which is defined by the separation $\delta E=63.3 \Gamma_{0}$ between the energies $E_{m M}$ of the transitions excited. This separation corresponds to a hyperfine field $B=33 \mathrm{~T}$ at the iron nuclei. Because of the finite sample thickness, the initial decay is about three times faster than natural as discussed previously, ${ }^{10}$ and the first minimum of the DB occurs at $\sim 270 \mathrm{~ns}$ as expected for a sample with effective thickness parameter $\xi_{m M}^{s}=1.95$ corresponding to a Mössbauer thickness $T=7.8$ for each $M-m=0$ transition.

The time evolution of NFS by an Invar foil of thickness $1.2 \mu \mathrm{m}$, which has an effective thickness parameter $\xi_{m M}^{s}$ $=1.4$ is shown in Fig. 3(b). Although the effective thickness is less than for the iron foil, the decay is much faster. It is approximately exponential with a decay constant 6.5 times larger than natural, and the signal is definitely lost after 150 $\mathrm{ns}$. The period of the QB is almost $16 \mathrm{~ns}$, which is larger than for iron metal, in agreement with the smaller hyperfine splitting observed in the Mössbauer spectra (compare Fig. 2).

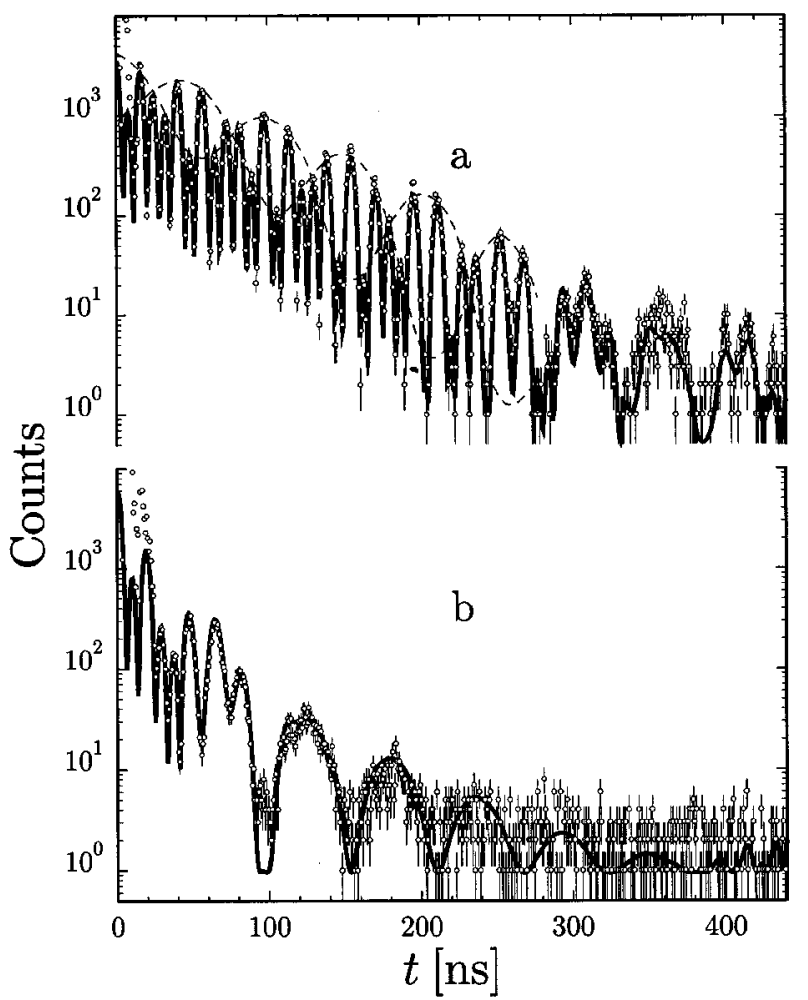

FIG. 4. Time evolution of NFS of SR for a $1 \mu \mathrm{m}$ iron metal foil (a) and a $1.2 \mu \mathrm{m}$ Invar foil (b) in a horizontal magnetic field of $\sim 0.13 \mathrm{~T}$. The solid lines are fits using the NFS theory. The dashed lines are guidelines to the eye, pointing out the $\sim 100$ ns modulation of the fast beat, starting high (low) at time zero for the even (uneven) numbered beats, respectively.

A more complicated beat pattern was observed when the samples were placed in a horizontal magnetic field. In this geometry the four nuclear transitions with $M-m= \pm 1$ are excited. The QB pattern observed in case of the thin iron foil is depicted in Fig. 4(a). We shall discuss it in Sec. V A. The corresponding time dependence for the $1.2 \mu \mathrm{m}$ Invar foil is shown in Fig. 4(b). Although the QB patterns are more complicated, compared to that in Fig. 3, they show the same basic difference: a faster decay of the signal in the time spectrum of Invar despite comparable effective resonance thicknesses.

\section{B. Thick samples}

The next step was to measure the NFS from thick foils to reveal the influence of the inhomogeneous broadening on the combination of interresonance and intraresonance interference. In a comprehensive study, the time dependences of NFS of Invar foils of different thicknesses and of the corresponding iron foils were measured for the two cases of transverse magnetization. Because of better evidence and greater simplicity, only the measurements in a vertical magnetic field, where the transitions with $M-m=0$ are excited alone, will be shown in the following.

As an example for the results obtained with the iron samples, Fig. 5(a) shows the time dependence of NFS by a foil of $\sim 9 \mu \mathrm{m}$ thickness. The fast QB is modulated by a pronounced slow DB, as previously reported. ${ }^{26}$ This case provides an example, where the approximate solution Eq. (16) holds. A strong initial speed-up of the decay leads immediately into the first DB minimum at $\sim 26 \mathrm{~ns}$. The period 


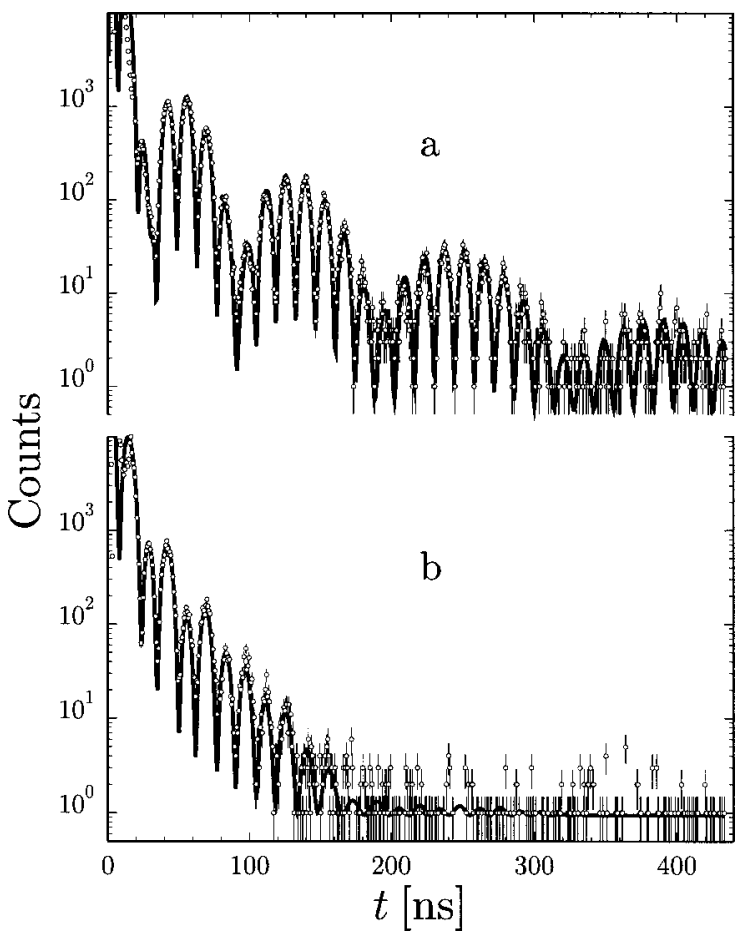

FIG. 5. Time evolution of NFS of SR for thicker foils in vertical magnetic fields. Iron metal foil of $\sim 9 \mu \mathrm{m}$ thickness at $4 \mathrm{~K}$ in a field of $1 \mathrm{~T}$ (a) and Invar foil of $\sim 10 \mu \mathrm{m}$ thickness at room temperature in a field of $0.13 \mathrm{~T}$ (b). The solid lines are fits using the NFS theory.

of the DB increases with time, ${ }^{8,3}$ and so the later DB minima appear with increasing distances at times $\sim 90,190$, and 320 ns.

By contrast, the time dependence of NFS of a corresponding Invar foil of $\sim 10 \mu \mathrm{m}$ thickness [Fig. 5(b)] showed three unexpected features: (1) The apparent QB has a shorter period $(\sim 14 \mathrm{~ns})$ than measured for the $1.2 \mu \mathrm{m}$ Invar foil $(\sim 16$ ns), (2) no modulation by a DB can be recognized, (3) instead the apparent QB shows a perceptible high-low modulation.

In order to study these surprising features in more detail, the time dependence of NFS of the $40 \mu \mathrm{m}$ Invar foil was measured [Fig. 6(a)]. All three anomalies listed above are observed again. The apparent QB has now a period of $12 \mathrm{~ns}$ only, corresponding to a hyperfine splitting larger than in iron metal, and much larger than given by the Mössbauer spectrum of this foil [Fig. 2(d)].

This compression of the apparent QB was observed so far in this study for foils of different thickness. In order to exclude any influences of metallurgical differences between various foils, the same foil was measured with the effective thickness being increased by tilting around a vertical axis. This way the conditions for nuclear excitation stayed the same (only the transitions with $M-m=0$ were excited), but the effective foil thickness was increased to 57 and $76 \mu \mathrm{m}$, respectively. The measured time dependences are shown in Figs. 6(b) and 6(c). The apparent QB is still more compressed, with the period decreasing to a value of only $11 \mathrm{~ns}$. A QB of this period would correspond to an inner magnetic field of more than $40 \mathrm{~T}$.

The absence of the DB could in principle be explained by thickness inhomogeneities which are known to smear out the sharp DB minima. In order to exclude this effect, the NFS

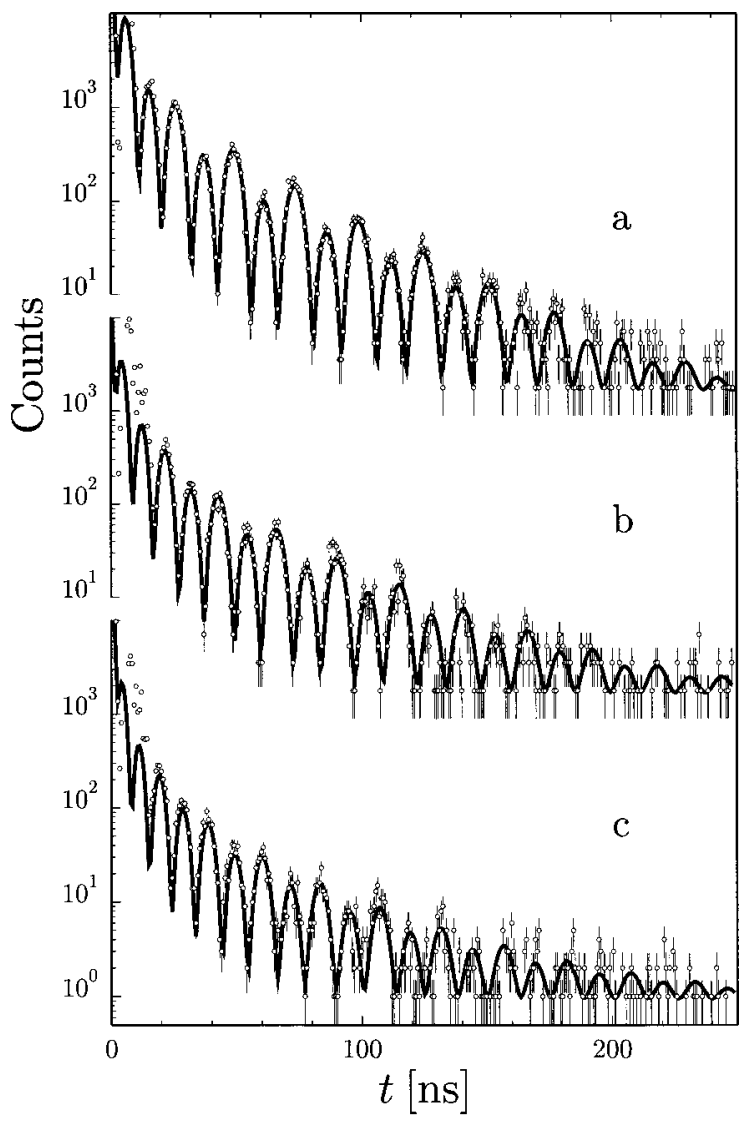

FIG. 6. Time evolution of NFS of SR for a $40 \mu \mathrm{m}$ Invar foil in a vertical magnetic field of $\sim 0.13 \mathrm{~T}$, mounted at right angle to the beam (a) and inclined by $\sim 45^{\circ}$ (b) and $\sim 58^{\circ}$ (c), yielding thicknesses of 40, 57, and $76 \mu \mathrm{m}$, respectively. The solid lines are fits using the NFS theory.

was measured from a thinner Invar foil of $6 \mu \mathrm{m}$ thickness, which should yield well separated and relatively broad DB minima. Figure 7(a) shows the time evolution of NFS by this foil. Again, the DB modulation, which should have a first minimum at $\sim 70 \mathrm{~ns}$, is completely missing.

At that time model considerations and fit results indicated, that the absence of the DB modulation might be connected with an asymmetry of the field distribution, which is typical for Invar at room temperature. ${ }^{18}$ At low temperatures, by contrast, the field distribution had been found to be much more symmetrical. ${ }^{18}$ In order to check the influence of different resonance shapes, the NFS of the same $6 \mu \mathrm{m}$ Invar foil was then measured at $4 \mathrm{~K}$ [Fig. 7(b)]. Indeed, the time dependence now reveals a DB envelope with rather pronounced minima around 50 and $150 \mathrm{~ns}$.

\section{INTERPRETATION AND DISCUSSION}

\section{A. Time spectra in the absence of inhomogeneous broadening}

In cases where two equivalent nuclear transitions with $M-m=0$ are excited, e.g., in the case of Figs. 3(a) and 5(a), the time spectra of NFS are very easy to "read" since they contain a QB pattern with a single frequency and a very distinct DB modulation which depends on the sample thickness. Already in cases when four nuclear transitions with 


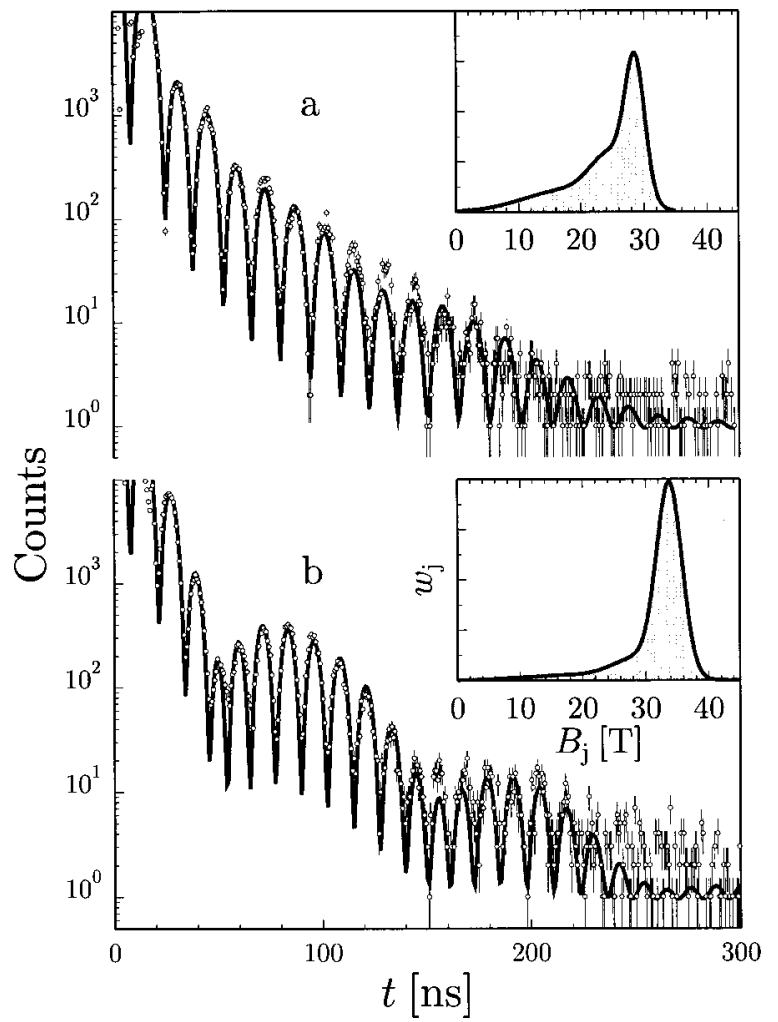

FIG. 7. Time evolution of NFS of SR for a $6 \mu \mathrm{m}$ Invar foil at room temperature in a vertical magnetic field of $\sim 0.13 \mathrm{~T}$ (a) and at $4 \mathrm{~K}$ in a vertical magnetic field of $1 \mathrm{~T}$ (b). The solid lines are fits using the NFS theory. The insets show the field distributions used for the fit.

$M-m= \pm 1$ are excited, the interpretation becomes less evident. Therefore we shall describe more precisely the relevant time spectrum shown in Fig. 4(a). For this discussion we shall use the approximation of a thin nuclear resonance scatterer. According to Eq. (14) we can write the expression for the NFS intensity

$$
\begin{aligned}
I(t) \propto & \exp \left(-\Gamma_{0} t / \hbar\right)\left[\cos ^{2}(\Omega t / 2)+\frac{2}{3} \cos (\Omega t / 2) \cos (\omega t / 2)\right. \\
& \left.+\frac{1}{9} \cos ^{2}(\omega t / 2)\right] .
\end{aligned}
$$

In this expression the first term corresponds to the interference of the two outer transitions in ${ }^{57} \mathrm{Fe}$ separated in energy by $\hbar \Omega$, the third term arises from the interference of the two inner transitions with energy separation $\hbar \omega$, and the second term describes the interference of the outer and the inner transitions. ${ }^{27}$ In accordance with Eqs. (5)-(8) the ratios of the thickness parameters $\xi_{m M}^{s}$ are 3:1:1:3.

In the time window from 0 to $270 \mathrm{~ns}$ [Fig. 4(a)] a fast beat with a period of $8.1 \mathrm{~ns}$ is dominant, which corresponds to the first term in the above expression. The third term can be neglected. The second term, however, introduces an interesting modulation of the fast beat. Similar to the case of nuclear Bragg diffraction, ${ }^{27}$ the interference of the four $M-m$ $= \pm 1$ transitions causes also in NFS a high-low pattern with changing order. The order depends on the factor $\cos (\omega t / 2)$, which changes sign every $51 \mathrm{~ns}$. When we number the fast beats, starting with zero at time zero, we can easily follow the $\sim 100$ ns modulation of the even beats and the corresponding antiphase modulation of the uneven beats [compare guidelines in Fig. 4(a)].

At later times, however, dynamical effects make the scattering by the outer transitions largely vanish in a time window centered at $\sim 360 \mathrm{~ns}$, which is the first minimum of the DB pattern for the outer transitions. In this time window the scattering from the inner transitions prevails, which is characterized by a slow QB with a period of $\sim 50 \mathrm{~ns}$. The dominance of the scattering by the inner transitions, however, is only temporary: At still later times the contribution of the outer transitions would recover and the fast beat would dominate again.

\section{B. Faster decay caused by resonance broadening}

The time dependences of NFS by the thin Invar foil [compare Figs. 3(b) and 4(b)] show much faster decays than those of the thin iron metal foil [compare Figs. 3(a) and 4(a)]. In particular, the Invar NFS signal is definitely lost after times of $\sim 300,150$, and $100 \mathrm{~ns}$ for the inner, middle, and outer pairs of transitions, respectively. This demonstrates in agreement with the results of Sec. II B, that the faster decay in case of the thin Invar foil is primarily due to inhomogeneous nuclear resonance broadening. In this case the radiation components are spread in frequency in a range wider than the natural linewidth, and therefore they run out of phase faster than in a natural lifetime.

A similar, faster decay of the NFS signal due to dephasing can be observed in case of dynamic inhomogeneous resonance broadening, for instance in cases of diffusion ${ }^{28}$ or relaxation. ${ }^{29}$ This raises the question, whether Mössbauer spectroscopy in time domain offers possibilities to distinguish between dynamic and static origins of inhomogeneous nuclear resonance broadening. In conventional Mössbauer spectroscopy a distinction between different origins of inhomogeneous broadening is not straightforward. It requires either measurements using the selective excitation double Mössbauer method (SEDM) $)^{30}$ or a special analysis of the line shapes. ${ }^{19}$ It seems, that in time-domain spectroscopy the answer is not straightforward as well.

\section{Hybrid beat caused by asymmetry of the resonance broadening}

It was possible to fit all NFS time evolutions ${ }^{14}$ of the Invar foils by assuming asymmetric magnetic field distributions. Such distributions are typical for Invar alloys. ${ }^{18}$ Following Ref. 18 we have modeled appropriate distributions by the superposition of three Gaussians of different weights $W_{i}$, positions $B_{i}$, and widths $\Delta B_{i}$ :

$$
w_{j}=\sum_{i=1,2,3} \frac{W_{i}}{\Delta B_{i} \sqrt{2 \pi}} \exp \left[-\frac{\left(B_{j}-B_{i}\right)^{2}}{2\left(\Delta B_{i}\right)^{2}}\right] .
$$

The weights $W_{i}$ were normalized, so that $\Sigma_{j} w_{j}=1$. Typically we used about 40 different nuclear sites in the simulations. The fit parameters $W_{i}, B_{i}, \Delta B_{i}$ were optimized. ${ }^{31}$ As an example, the resulting profiles used for the fit of the $6 \mu \mathrm{m}$ 


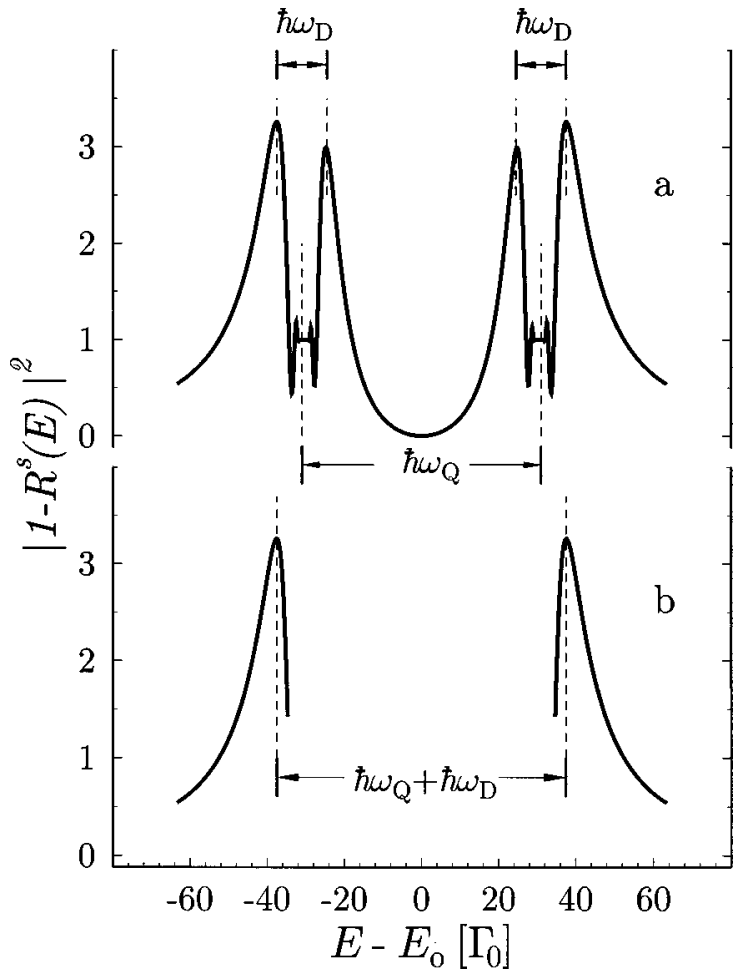

FIG. 8. Energy dependence of the NFS intensity in case of an optically thick scatterer with two resonance lines separated by a large hyperfine splitting. Due to multiple scattering, each resonance shows the typical double-hump structure. The hyperfine splitting is given by $\hbar \omega_{Q}$, the separation of the two humps by $\hbar \omega_{D}$ (a). This situation would yield a time evolution of NFS characterized by a fast QB with frequency $\omega_{Q}$, modulated by a DB with a frequency (decreasing in time) of order of $\omega_{D}$. (b) When the two inner humps are canceled, a hybrid beat with frequency $\omega_{Q}+\omega_{D}$ is expected.

Invar foil at room temperature and at $4 \mathrm{~K}$ are shown as insets in Figs. 7(a) and 7(b), respectively.

The important question is, why an asymmetric nuclear resonance broadening causes such drastic effects in NFS. An answer can be found if details of the energy spectrum of NFS are considered. In NFS of SR in thick samples, multiple scattering leads in the energy dependence of the forward scattered intensity to a so called double-hump structure at each resonance transition. ${ }^{3}$ Figure 8 shows a typical NFS energy spectrum $\left|1-R^{s}(E)\right|^{2}$ resulting from the excitation of two nuclear transitions at $E=E_{0} \pm \hbar \omega_{Q} / 2 . R^{s}(E)$ is calculated according to Eq. (9). The separation $\hbar \omega_{Q}$ of the centers of the two double humps determines the $\mathrm{QB}$ arising from inter-resonance interference, and the separation $\hbar \omega_{D}$ of the humps, which increases with sample thickness, causes the DB. If we now imagine a mechanism, that would just cancel the inner ones of each pair of humps, we immediately obtain an explanation for the anomalous features observed in case of the thick Invar foils [compare Fig. 8(b)]: DB and QB cease to exist separately, and instead blend into a new hybrid beat, which originates from the interference of the radiation components belonging to the two outer humps, with a beat frequency given approximately by the sum of $\omega_{Q}$ and $\omega_{D}$. Thus the DB would disappear, and the period of the new hybrid beat would decrease with increasing sample thickness, as observed in the experiment.

Indeed, interference of neighboring oscillators in an

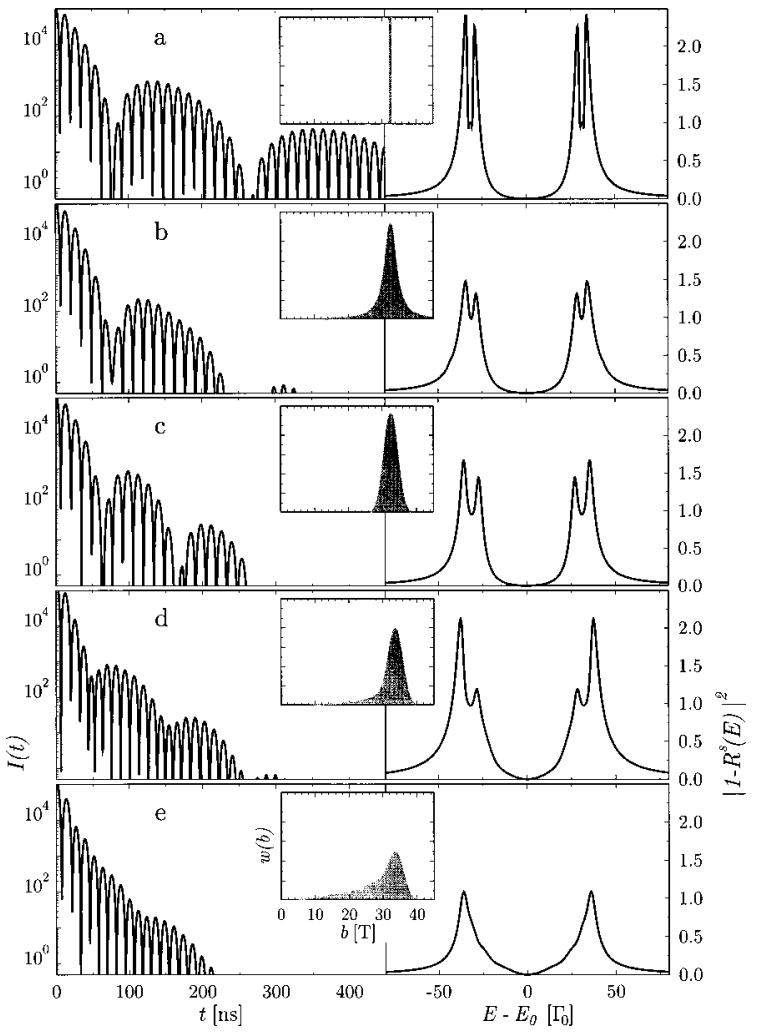

FIG. 9. Time evolution of NFS (left), energy dependence of NFS (right) and field profile (center) for different magnetic field distributions: definite value of magnetic field (a), symmetrical distributions of Lorentzian shape (b) and of Gaussian shape (c), slightly asymmetrical distribution corresponding to Invar at $4 \mathrm{~K}$ (d), and strongly asymmetrical distribution (e) based on Invar at room temperature.

asymmetrical oscillator distribution is just such a mechanism. Figure 9 shows the effect of resonance broadening on the two double-hump structures and on the corresponding time evolution of NFS. In Fig. 9(a) a definite value was assumed for the magnetic field, as in the case of iron metal for instance. The resulting double-hump structures are well shaped, and in the time spectrum a fast QB is modulated by a pronounced DB. In Figs. 9(b) and 9(c) symmetrical distributions of Lorentzian shape and of Gaussian shape, respectively, were assumed. The general double-hump character and the resulting strong modulation of the time spectrum by a DB remain essentially unchanged. In addition, we want to point out that the interference of neighboring oscillators in an asymmetrical oscillator distribution is a more general phenomenon, which is, e.g., also observed in x-ray physics. ${ }^{32}$

A fundamental change, however, occurs, when an asymmetry is introduced to the field distribution. In case of asymmetric broadening, with oscillator densities which decrease slower in the region between the lines than outside of them, the inner humps are much stronger affected by destructive interference than the outer ones [see Fig. 9(d)]. In the case of a pronounced asymmetry, they can even be completely cancelled [see Fig. 9(e)]. These hump structures then yield NFS time evolutions with less pronounced or finally completely missing DB modulations [Figs. 9(d) and 9(e)]. Note that the field distribution of Fig. 9(d) is actually the one used for the fit of the NFS by the $6 \mu \mathrm{m}$ Invar foil measured at $4 \mathrm{~K}$ 
[compare Fig. 7(b)]. The field distribution of Fig. 9(e) is the one used for the fit of the NFS of the same foil measured at room temperature [compare Fig. 7(a)], however with the positions $B_{i}$ of the three Gaussians being scaled so that the highest position corresponds to the one of Figs. 9(a) -9 (d).

The resulting hybrid beat was found to be extremely sensitive to details of the field distribution. The rather smooth slope observed in Fig. 7(a) seems to be accidental, in general the hybrid beat is modulated by a high-low pattern as observed in Fig. 5(b) and Fig. 6. Such a high-low pattern arises, whenever the beat of two dominating radiation components is disturbed by additional frequency components. Thus the high-low modulation reveals details of the frequency spectrum of the scattered radiation. This frequency spectrum is determined by the interference of the scattering from the oscillator distribution, which reflects the actual field distribution in the sample.

It should be noted, that already in the case of a thin foil [see Fig. 3(b)] the same interference effects lead to a sharp $\mathrm{QB}$, in spite of a pronounced asymmetry of the oscillator distribution [compare the room-temperature field distribution depicted in Fig. 7(a)]. Also in the kinematical scattering by thin foils destructive interference largely suppresses the contribution of the oscillators at the inner slopes of their energy distribution. This leads in the energy dependence of the scattering to peaks, which are sharper, more symmetrical and slightly shifted outwards as compared to the corresponding strongly asymmetrical dips of the absorption spectra. Such an energy dependence of the scattering yields a sharp QB with slightly increased frequency.

Noteworthy, a similar, but slow hybrid between QB's and DB's arises already in the absence of resonance broadening, when the hyperfine splitting is so small, that it is in the order of the energy separation of the double humps. In this situation the interference of the forward scattering amplitudes is destructive in between the split resonances, and constructive outside. This leads again to a two-hump structure as the one depicted in Fig. 8(b), however, with much smaller separation of the outer humps, yielding a slow beat. Such a slow hybrid beat is quite common for NFS by thick samples, which exhibit, e.g., quadrupole splitting. The slow hybrid beat is presently under study.

\section{CONCLUSION}

In the time evolution of NFS by thin Invar foils a fast decay of the scattering signal was observed. It originates from dephasing in time, which is caused by inhomogeneous nuclear-resonance broadening. In the case of thick Invar foils, a strong influence of the asymmetry of the resonance broadening on the time response was observed, which in particular leads to a blend of QB and DB into a fast hybrid beat.

The fast hybrid beat is typical for large hyperfine splittings in case of asymmetric magnetic field distributions. Since it has a shorter period than the original QB, it would yield erroneous values for the hyperfine splitting, if it is misinterpreted as a pure QB. This again demonstrates that multiple scattering in case of a coherent scattering channel such as NFS plays an important role. The fast hybrid beat revealed itself as being very sensitive to the particular shapes of the magnetic field distribution in Invar. In the present paper the inhomogeneous broadening was modeled with static magnetic field distributions, where the nuclei of the ensemble experience definite, but slightly different magnetic fields.

\section{ACKNOWLEDGMENTS}

This work was funded by the Bundesministerium für Bildung, Wissenschaft, Forschung und Technologie under Contract Nos. 05 5WOAAI/643WOA and 05 643GUA. U.v.B. and G.V.S. would like to thank S. L. Ruby, J. Arthur, A. Q. R. Baron, G. S. Brown, and A. I. Chumakov for hospitality and collaboration in the preliminary experiments at the SSRL.
${ }^{1}$ E. Gerdau, R. Rüffer, H. Winkler, W. Tolksdorf, C. P. Klages, and J. P. Hannon, Phys. Rev. Lett. 54, 835 (1985).

${ }^{2}$ E. Gerdau and U. van Bürck, in Resonant Anomalous X-ray Scattering, Theory and Applications, edited by G. Materlik, C. J. Sparks, and K. Fischer (North-Holland, Amsterdam, 1994), p. 589.

${ }^{3}$ G. V. Smirnov, Hyperfine Interact. 97/98, 551 (1996); in $X$-ray and Inner-Shell Processes, edited by R. L. Johnson, H. SchmidtBöcking, and B. F. Sonntag, AIP Conf. Proc. No. 389 (AIP, New York, 1997), p. 323.

${ }^{4}$ J. B. Hastings, D. P. Siddons, U. van Bürck, R. Hollatz, and U. Bergmann, Phys. Rev. Lett. 66, 770 (1991).

${ }^{5}$ G. T. Trammell and J. P. Hannon, Phys. Rev. B 18, 165 (1978); 19, 3835(E) (1979).

${ }^{6}$ E. Gerdau, R. Rüffer, R. Hollatz, and J. P. Hannon, Phys. Rev. Lett. 57, 1141 (1986).

${ }^{7}$ F. J. Lynch, R. E. Holland, and M. Hamermesh, Phys. Rev. 120, 513 (1960).

${ }^{8}$ Yu. Kagan, A. M. Afanas'ev, and V. G. Kohn, J. Phys. C 12, 615 (1979).
${ }^{9}$ Yu. V. Shvyd'ko, S. L. Popov, and G. V. Smirnov, Pis'ma Zh. Éksp. Teor. Fiz. 53, 217 (1991) [JETP Lett. 53, 231 (1991)]; J. Phys.: Condens. Matter 5, 1557 (1993); 5, 7047 (1993).

${ }^{10}$ U. van Bürck, D. P. Siddons, J. B. Hastings, U. Bergmann, and R. Hollatz, Phys. Rev. B 46, 6207 (1992).

${ }^{11}$ Yu. V. Shvyd'ko, T. Hertrich, U. van Bürck, E. Gerdau, O. Leupold, J. Metge, H. D. Rüter, S. Schwendy, G. V. Smirnov, W. Potzel, and P. Schindelmann, Phys. Rev. Lett. 77, 3232 (1996).

${ }^{12}$ Yu. V. Shvyd'ko (unpublished).

${ }^{13}$ Yu. V. Shvyd'ko, G. V. Smirnov, S. L. Popov, and T. Hertrich, Pis'ma Zh. Éksp. Teor. Fiz. 53, 69 (1991) [JETP Lett. 53, 69 (1991)].

${ }^{14}$ MOTIF.23, a program for fitting time spectra of nuclear resonance forward scattering, available from the anonymous ftp:// i2aix04.desy.de/pub/motif/ or from the author via electronic mail: Yuri.Shvydko@desy.de. The User guide to MOTIF is the Postscript file motif_23.ps.

${ }^{15}$ E. Ikonen, P. Helistö, T. Katila, and K. Riski, Phys. Rev. A 32, 2298 (1985)

${ }^{16}$ Yu. V. Shvyd'ko and G. V. Smirnov, Nucl. Instrum. Methods Phys. Res. B 51, 452 (1990). 
${ }^{17}$ G. V. Smirnov and V. G. Kohn, Phys. Rev. B 52, 3356 (1995).

${ }^{18}$ J. Y. Ping, D. G. Rancourt, and R. A. Dunlap, J. Magn. Magn. Mater. 103, 285 (1992).

${ }^{19}$ E. Yu. Tsymbal, A. M. Afanas'ev, M. Fricke, and J. Hesse, Hyperfine Interact. 93, 1543 (1994).

${ }^{20}$ M. Dubé, P. R. L. Heron, and D. G. Rancourt, J. Magn. Magn. Mater. 147, 122 (1995); M.-Z. Dang, M. Dubé, and D. G. Rancourt, ibid. 147, 133 (1995).

${ }^{21}$ Preliminary studies were performed at the wiggler beamline 10-2 of the Stanford Synchrotron Radiation Laboratory. However, the quality of the data was not sufficient to allow an interpretation at that time.

${ }^{22}$ R. Rüffer, D. Giesenberg, H. D. Rüter, R. Hollatz, E. Gerdau, J. Metge, K. Ruth, W. Sturhahn, M. Grote, and R. Röhlsberger, Hyperfine Interact. 58, 2467 (1990).

${ }^{23}$ O. Leupold, E. Gerdau, H. D. Rüter, W. Meyer-Klaucke, A. X. Trautwein, and H. Winkler (unpublished).

${ }^{24}$ The high resolution monochromator with channel-cut $\operatorname{Si}\left(\begin{array}{lll}4 & 2 & 2\end{array}\right)$ and $\operatorname{Si}\left(\begin{array}{lll}1 & 2 & 2\end{array}\right)$ crystals in the nested geometry was designed by E. Gerdau, R. Rüffer, and H. D. Rüter based on the proposal of T. Ishikawa, Y. Yoda, K. Izumi, C. K. Suzuki, X. W. Zhang, M. Ando, and S. Kikuta, Rev. Sci. Instrum. 63, 1015 (1992).

${ }^{25}$ A. Q. R. Baron, Nucl. Instrum. Methods Phys. Res. A 352, 665 (1995)

${ }^{26}$ S. Kikuta, in Resonant Anomalous X-ray Scattering, edited by G. Materlik, C. J. Sparks, and K. Fischer (North-Holland, Amsterdam, 1994), p. 635.

${ }^{27}$ U. van Bürck, R. L. Mössbauer, E. Gerdau, R. Rüffer, R. Hollatz, G. V. Smirnov, and J. P. Hannon, Phys. Rev. Lett. 59, 355 (1987).
${ }^{28}$ B. Sepiol, A. Meyer, G. Vogl, R. Rüffer, A. I. Chumakov, and A. Q. R. Baron, Phys. Rev. Lett. 76, 3220 (1996).

${ }^{29}$ H. Winkler, O. Leupold, S. Schwendy, W. Meyer-Klaucke, H. D. Rüter, and A. X. Trautwein (unpublished).

${ }^{30}$ D. C. Price and A. M. Stewart, Hyperfine Interact. 42, 1157 (1988).

${ }^{31}$ Actually, the optimal fit parameters for the time dependences of NFS by different foils varied slightly. This can easily be explained by minor metallurgical changes introduced due to different heat treatment in the preparation process, in particular by the annealing in the course of rolling. The thicknesses of the foils were sufficiently homogeneous so that in all fits averaging over thickness variations proved to be unnecessary.

${ }^{32}$ In x-ray physics, the peculiar shape of the refractive index near an atomic edge is caused by asymmetric resonance broadening [R. W. James, The Optical Principles of the Diffraction of X-rays (G. Bell and Sons, London, 1958), Chap. IV.1.(k)]. Near an atomic edge, the atomic oscillator distribution, as obtained by $\mathrm{x}$-ray absorption measurements, is extremely asymmetric, resembling a saw-tooth distribution. For each oscillator of this distribution, the scattering amplitude is negative above and positive below resonance. Within the distribution, the scattering amplitudes above the individual resonances are almost cancelled by corresponding amplitudes of opposite sign belonging to neighboring resonances of slightly higher energy. As a result, only a small positive amplitude below the atomic edge remains. The essential point is, that the real part of resonant scattering, which changes sign at resonance, is extremely sensitive to the particular distribution of oscillators. 